\title{
Fever of unknown origin caused by intrathyroidal thymic carcinoma
}

\author{
Seung Min Chung ${ }^{1}$, Kyung-Ju Kim² ${ }^{2}$, Jun Sung Moon¹, Young Hoon Hong? ${ }^{3}$, and Su Hwan Kang ${ }^{4}$
}

${ }^{1}$ Division of Endocrinology and Metabolism, Department of Internal Medicine, ${ }^{2}$ Department of Pathology, ${ }^{3}$ Division of Rheumatology and Infectious Disease, Department of Internal Medicine, ${ }^{4}$ Department of Surgery, Yeungnam University College of Medicine, Daegu, Korea
Received: July 4, 2017

Revised : October 8, 2017

Accepted: October 8, 2017

\section{Correspondence to \\ Jun Sung Moon, M.D. \\ Tel: $+82-53-620-3825$ \\ Fax: +82-53-654-8386 \\ E-mail:mjs7912@yu.ac.kr}

A 44-year-old man with a 1-month history of fever visited the department of infectious disease. His temperature was $38.4^{\circ} \mathrm{C}$ and non-movable, non-tender mass with firm consistency was palpated on right thyroid gland. Laboratory tests showed elevated levels of C-reactive protein $(15.6 \mathrm{mg} / \mathrm{dL})$ and erythrocyte sedimentation rate $(85$ $\mathrm{mm} / \mathrm{hr}$ ), but normal range of white blood cell count $\left(9,750 / \mathrm{mm}^{3}\right)$, thyroid stimulation hormone (1.67 mIU/L), free thyroxine (12.28 pmol/L), and thyroglobulin $(5.30 \mathrm{ng} / \mathrm{mL})$. Blood and urine cultures were sterile. Positron emission tomography-computed tomography showed a high fluorodeoxyglucose uptake on the lower pole of the right thyroid gland (Fig. 1A). Thyroid ultrasound revealed $3.3 \times 2.5 \times 2.3 \mathrm{~cm}$ sized hypoechoic, heterogeneous mass with irregular margin on right lobe (Fig. 1B) and its cytology contained some atypical cell clusters with prominent nucleoli. Within core needle bi- opsy, the solid nests of polygonal cells were separated by fibrous bands with many lymphocytes infiltration (Fig. 2A). The tumor cells had either ill-defined cell border with vesicular and prominent nucleoli, or had distinct cell border with focal squamoid feature (Fig. $2 \mathrm{~B}$ and $2 \mathrm{C}$ ). The immunohistochemistry staining showed diffuse positivity for $\mathrm{CD}_{117}$, CD5, p63 (Fig. 2D$2 \mathrm{~F}$ ), and negative for TTF1, calcitonin, and synaptophysin. The patient was diagnosed as intrathyroidal thymic carcinoma (ITC). Total thyroidectomy and adjuvant radiotherapy (6o Gray/30 fraction) was performed. After the operation, he was no more feverish.

ITC is a rare malignant epithelial tumor of the thyroid gland with thymic epithelial differentiation arising from ectopic thymus or branchial pouch remnants. Common symptoms are neck mass and hoarseness but rarely, thymic carcinoma can also cause fever. Immunohistochemistry plays an im-
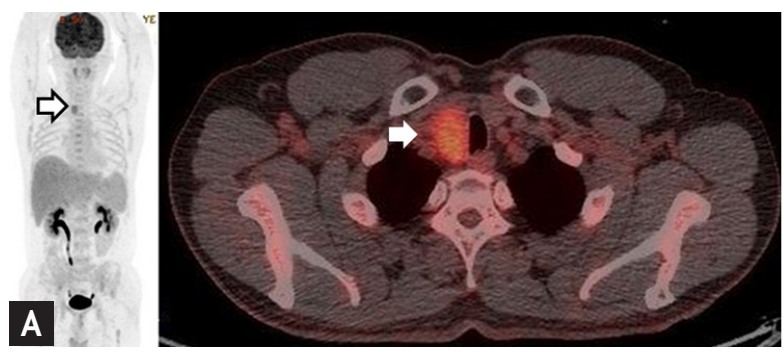

Figure 1. (A) Positron emission tomography-computed tomography revealed high fluorodeoxyglucose uptake on lower pole of right thyroid gland (arrows) and (B) thyroid ultrasonogram revealed $3.3 \times 2.5 \times 2.3 \mathrm{~cm}$ sized hypoechoic, heterogeneous mass with irregular margin on lower pole of right thyroid gland (arrow). 

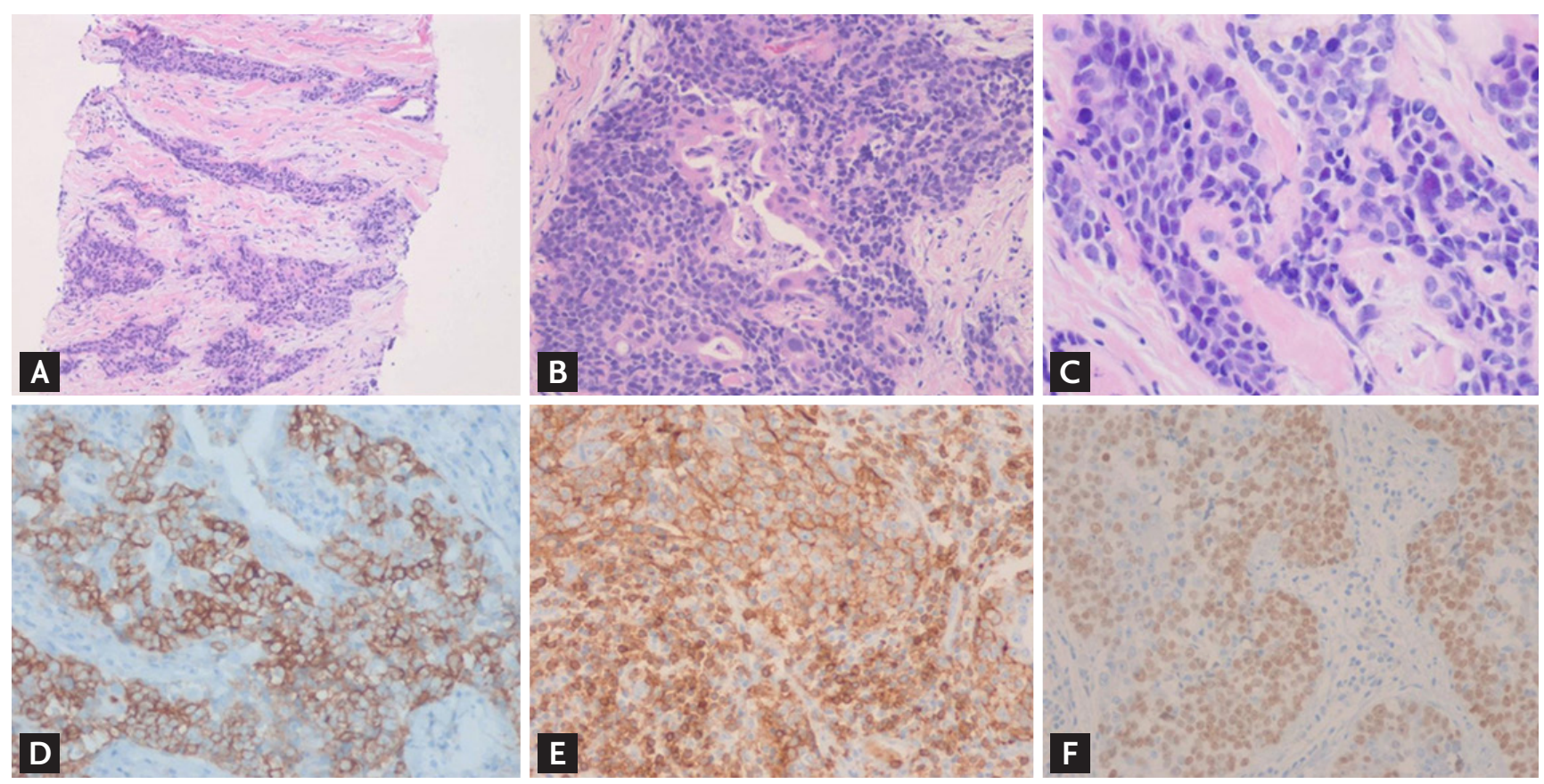

Figure 2. (A) The tumors were arranged in solid nests and separated by fibrous stroma (H\&E, ×100). (B) The tumor cells had polygonal shaped nuclei and focal squamous differentiation (H\&E, $\times 200)$. (C) The tumor cell nuclei had vesicular chromatin and prominent nucleoli (H\&E, $\times 400)$. Immunohistochemistry staining showed a diffuse positive for (D) CD117 ( $\times 200),(\mathrm{E}) \mathrm{CD}_{5}$ $(\times 200)$, and $(F)$ p63 $(\times 200)$.

portant role in distinguishing ITC from other thyroid malignancies. The positivity for $\mathrm{CD}_{5}$ is a key feature. It has better prognosis than either anaplastic or squamous cell thyroid carcinoma and curative surgery is the therapeutic choice.

Written informed consents were obtained.

\section{Conflict of interest}

No potential conflict of interest relevant to this article was reported. 